\title{
Influence of lysosomal sequestration on multidrug resistance in cancer cells
}

\author{
Reginald Halaby \\ Department of Biology, Montclair State University, Montclair, NJ 07043, USA.
}

Correspondence to: Dr. Reginald Halaby, Department of Biology, Montclair State University, 1 Normal Ave, Montclair, NJ 07043, USA. E-mail: halabyr@montclair.edu

How to cite this article: Halaby R. Influence of lysosomal sequestration on multidrug resistance in cancer cells. Cancer Drug Resist 2019;2:31-42. http://dx.doi.org/10.20517/cdr.2018.23

Received: 2 Nov 2018 First Decision: 5 Nov 2018 Revised: 15 Jan 2019 Accepted: 24 Jan 2019 Published: 19 Mar 2019

Science Editor: Godefridus J. Peters Copy Editor: Cui Yu Production Editor: Huan-Liang Wu

\begin{abstract}
Chemotherapy remains a primary treatment modality for various malignancies. However, resistance to chemotherapeutic drugs is a major obstacle to curative cancer therapy. Lysosomes are acidic organelles that participate in cellular digestion. However, there is rising interest in lysosomes because of their involvement with cancer. For example, extracellular secretion of lysosomal enzymes promote tumorigenesis; cytosolic leakage of lysosomal hydrolases promote apoptosis; and weak chemotherapeutic bases diffuse across the lysosomal membrane and become entrapped in lysosomes in their cationic state. Lysosomal drug sequestration lowers the cytotoxic potential of chemotherapeutics, reduces drug availability to sites of action, and contributes to cancer resistance. This review examines various mechanisms of lysosomal drug sequestration and their consequences on cancer multidrug resistance. Strategies for overcoming drug resistance by exploiting lysosomes as subcellular targets to reverse drug sequestration and drug resistance are also discussed.
\end{abstract}

Keywords: Lysosomal sequestration, lysosomes, chemotherapeutics, multidrug resistance, cancer, permeabilityglycoprotein, exocytosis

\section{INTRODUCTION}

Lysosomes are acidic organelles that contain over 50 digestive enzymes that can degrade all macromolecules. They are the major cell digestive organelles ${ }^{[1]}$. In addition to this housekeeping function, lysosomes perform diverse cellular processes. For example, they are involved in macroautophagy, chaperone-mediated autophagy, cholesterol homeostasis, and degrading receptor tyrosine kinase receptors and growth factors ${ }^{[2-6]}$. Lysosomal hydrolases play opposing roles in neoplastic cells. Lysosomal proteases that are secreted

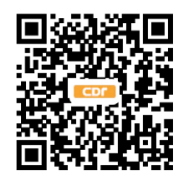


extracellularly promote tumor invasion and metastasis ${ }^{[7-9]}$. Specifically, cathepsins are used by tumor cells to degrade extracellular matrix components such as fibronectin, elastin, and laminin, thereby facilitating invasion, angiogenesis, and metastasis ${ }^{[10,11]}$. In contrast, cytosolic translocation of lysosomal proteases induces apoptosis and cell death ${ }^{[12,13]}$. Due to the dual role that lysosomes have in cancer, mounting evidence suggests that they are attractive targets for oncological therapies ${ }^{[14,15]}$.

Drug resistance is the primary reason that cancer treatments fail and patients die. Multidrug resistance (MDR) occurs in cancer cells that develop the ability to resist various drugs that are structurally and pharmacologically unrelated ${ }^{[16]}$. It is well documented that the drug transporter permeability-glycoprotein (P-gp) contributes to $\mathrm{MDR}^{[17]}$. Al-Akra and his team demonstrated that lysosomal P-gp plays an important role in conferring drug resistance ${ }^{[18]}$. Additionally, P-gp has been observed to localize to lysosomes ${ }^{[19]}$. In spite of novel approaches to address MDR, it continues to be a major cause of unsuccessful cancer treatments ${ }^{[20,21]}$.

Mounting evidence shows that lysosomes play a role in MDR. A major limitation of chemotherapeutic drugs is that they become trapped or sequestered in acidic organelles, such as the lysosomes. Lipophilic chemotherapeutic agents with weak base properties readily diffuse across cell membranes. However, when these drugs enter the acidic lumen of lysosomes, they become protonated and are trapped in the lysosomes ${ }^{[22]}$. Mounting evidence has demonstrated that certain hydrophobic weak base chemotherapeutics such as the anthracyclines doxorubicin, daunorubicin, and mitoxantrone, imidazoacridinones, the receptor tyrosine kinase inhibitor sunitinib, and pyrimethamine preferentially accumulate in lysosomes ${ }^{[23-30]}$. Lysosomal sequestration markedly reduces drug concentrations and thereby directly decreases the effects of anticancer drugs on their intended targets, the nucleus and cytoplasm. This lysosomal scavenging of oncologic drugs potentiates MDR. Therefore, understanding the molecular mechanism underlying lysosomal sequestration of chemotherapeutic drugs should provide insights to circumvent this clinical problem.

\section{LYSOSOMAL MEMBRANE PERMEABILIZATION}

Lysosomes can also initiate the intrinsic apoptosis pathway in response to treatment by lysosomotropic agents. Li et al $^{[31]}$ (2000) were one of the first groups to demonstrate that lysosomotropic agents disrupted the lysosomal membrane, resulting in cytosolic leakage of the acid hydrolases, specifically the cathepsins, and apoptosis. Subsequently, various studies have confirmed that lysosomal membrane permeabilization (LMP) induces apoptosis ${ }^{[12,32,33]}$.

Since LMP is known to trigger apoptosis in various cancer cells, drug screens to identify agents that induce LMP may prove to be effective cancer therapies to overcome drug resistance induced by lysosomal drug sequestration. For example, a recent study detected 175 compounds that induced death in HCT116 colon cancer cells. Notably, over half of the 11 compounds that induced apoptosis in p-53 deficient cells did so by LMP and cathepsin-mediated cell death ${ }^{[34]}$. Additionally, the hydrophobic weak base siramesine induced LMP in cancer cells in vitro and in vivo ${ }^{[35,36]}$. Consistent with this approach, bovine $\alpha$-lactalbumin and oleic acid was shown to kill various cancer cell lines (L1210 leukemia, HeLa cervical adenocarcinoma, PC-3 prostate adenocarcinoma, U118 MG glioblastoma, MCF-7 breast adenocarcinoma and others) by a mechanism involving $\mathrm{LMP}^{[37]}$.

\section{CANCER CELLS MODIFY LYSOSOMES TO EVADE CELL DEATH}

The success of cancer cells to develop resistance to chemotherapeutics also involves mutating pro-apoptotic pathways and lysosomal-mediated death pathways while upregulating cell proliferation pathways. Tumor cells use various methods to modify their lysosomes in an effort to evade cell death. The increased activity of phosphatidylinositol-3'-kinase (PI3K), which is characteristic of many tumors ${ }^{[38-40]}$, promotes stability in 
tumor cell lysosomes. Mousavi et al. ${ }^{[41]}$ (2003) reported that PI3K regulates the size, maturation, and activity of lysosomes. Tumor cells can abolish LMP by overexpression of cytosolic protease inhibitors ${ }^{[42,43]}$. Cancer cells also protect themselves from LMP by translocating Hsp70 from the cytosol to the lysosomal lumen where it stabilizes lysosomal membranes by promoting the activity of acid sphingomyelinase ${ }^{[44,45]}$. Support for this role of Hsp70 comes from observations that depletion of this protein triggers a tumor-cell-specific lysosomal cell death program ${ }^{[46]}$.

\section{LYSOSOMAL INVOLVEMENT IN MDR}

\section{Lysosomal sequestration of weak bases}

Lysosomes have been shown to sequester lipophilic, weakly basic chemotherapeutic drugs via a nonenzymatic and non-transporter mediated mechanism ${ }^{[23]}$. Notably, adriamycin was observed to concentrate in the lysosomes of drug-resistant cells but not in lysosomes drug-sensitive cells ${ }^{[47]}$. Presumably these weak bases are freely transported to the lysosomes by passive transport due to their hydrophobic composition. The pKa values, predominantly above 7.0, for these drugs confirms that they are weak bases. Likewise, the intracellular efficacy of these compounds can be decreased in vitro at acidic $\mathrm{pH}^{[23,25]}$. Many of the drugs used to treat malignancies are weak bases, and it has been demonstrated by several reports that they become sequestered in lysosomes. These include the following drugs: daunorubicin, doxorubicin, lapatinib, vincristine, and nintedanib ${ }^{[23,25,27,36,48]}$. Once they cross the lysosomal membrane, these weak base chemotherapeutics become trapped through protonation in the lysosomal lumen ${ }^{[4,50]}$. The use of the term "drug trapping" in lysosomes can be misleading. The ionized form, in most cases, is in equilibrium with the neutral drug in the cytosol. Furthermore, the ionized version of the drug can rapidly cross the lysosomal membrane by passive diffusion when the cytosolic concentration of the drug decreases due to transport into the bile, metabolism, or diffusion back into the plasma ${ }^{[23]}$. Support for this comes from the observation of rapid reversibility of lysosomal trapping of a lipophilic drug seen when rats are asphyxiated with carbon dioxide, which modestly acidifies the blood and causes a decrease in tissue levels and an increase in plasma drug levels ${ }^{[51]}$. These results suggest that lysosomes function as a reservoir pulling the drug from its target site and do not indefinitely trap cytotoxic drugs.

In contrast, localization of chemotherapeutics to the acidic lumen of lysosomes does promote MDR. Support for this comes from several lines of evidence. Lysosomal accumulation of sunitinib was detected in hepatocellular carcinoma cells, renal cancer cells, and colon cancer cells ${ }^{[24,52]}$. The multikinase inhibitor nintedanib has been investigated in clinical trials for the following tumors: non-small cell lung cancer, colorectal cancer, prostate cancer, and pancreatic cancer ${ }^{[53-56]}$. One study reported that nintedanib was sequestered in lysosomes, thus lowering its cytosolic concentrations and its fibroblast growth factor receptor inhibition potential ${ }^{[48]}$. Another report found that intracellular levels of imatinib are primarily determined by lysosomal sequestration ${ }^{[57]}$. Lysosomes have also been reported to have indirect effects on drug sequestration. Kalayda et al. ${ }^{[58]}$ reported that abnormalities in the lysosomal compartment promote sequestration of cisplatin away from the nucleus due to faulty localization of transport proteins. Taken together, these data suggest that anticancer drug resistance is modulated by lysosomal sequestration, as summarized in Table 1 . Further studies are warranted to determine whether targeting lysosomes can overcome resistance to chemotherapeutics.

Chemotherapeutic drugs are distributed between cytosolic and nuclear compartments intracellularly. Since lysosomes are not the intended target sites of these drugs, their entrapment in lysosomes effectively decreases their therapeutic effects at the wild type targets, such as nuclear DNA. Support for this notion comes from a study that showed that daunorubicin accumulation in lysosomes resulted in decreased nuclear concentrations of daunorubicin and drug resistance ${ }^{[25]}$. Likewise, another study found that lysosomal sequestration of doxorubicin in MCF-7/adriamycin breast cancer cells decreased the levels of the drug in the nucleus ${ }^{[4]}$. Indeed, it was reported that cells with a higher number of lysosomes were more resistant to 
Table 1. Chemotherapeutics that are sequestered in lysosomes and confer drug resistance

\begin{tabular}{llc}
\hline Drug & Molecular target & References \\
\hline Doxorubicin & Topoisomerase II inhibitor & {$[25]$} \\
Vinblastine & Antimicrotubule agent & {$[68]$} \\
Vincristine & Antimicrotubule agent & {$[70]$} \\
Methotrexate & Dihydrofolate reductase & {$[29]$} \\
Sunitinib & VEGFR2, PDGFRb, c-kit & {$[24]$} \\
Pyrimethamine & Dihydrofolate reductase & {$[30]$} \\
Lapatinib & EGFR, HER2 & {$[23]$} \\
Gefitinib & EGFR & {$[23]$} \\
Sorafenib & RAF, VEGFR & {$[52]$} \\
Nintedanib & VEGFR, FGFR, PDGFR & {$[48]$} \\
Topotecan & Topoisomerase I inhibitor & {$[83]$} \\
Imatinib & BCR-ABL & {$[48]$} \\
Pazopanib & VEGFR, PDGFR & {$[64]$} \\
Erlotinib & EGFR & {$[64]$} \\
\hline
\end{tabular}

VEGFR: vascular endothelial growth factor receptor; PDGFRb: beta-type platelet-derived growth factor receptor; EGFR: endothelial growth factor receptor; HER: human epidermal growth factor receptor; FGFR: fibroblast growth factor receptor; RAF: rapidly accelerated fibrosarcoma

sunitinib compared to cells with lower numbers of lysosomes ${ }^{[49]}$. Conflicting evidence comes from a study showing a cisplatin-resistant ovarian cancer cell line containing considerably fewer lysosomes than wildtype cells ${ }^{[59]}$.

\section{Lysosomal biogenesis and MDR}

Lysosomal biogenesis is typically a response to cell stress and is regulated by the translocation of transcription factor EB (TFEB) from the cytosol to the nucleus ${ }^{[60]}$. TFEB activity appears to be regulated through its phosphorylation by mammalian target of rapamycin complex 1 (mTORC1). mTORC1 was shown to exert its kinase activity on lysosomal surfaces where it phosphorylates TFEB, thereby inactivating the transcription factor ${ }^{[6]]}$. TFEB can be dephosphorylated by calcineurin, which transforms it to its active form and facilitates its nuclear translocation ${ }^{[62]}$. TFEB-mediated lysosomal biogenesis is induced by various stimuli, namely cell starvation, inhibition of mTORC1, and abnormal lysosomal storage ${ }^{[60]}$. Interestingly, lysosomal stress also modulates lysosomal gene expression ${ }^{[63]}$. A recent report showed that doxorubicin and mitoxantrone triggers TFEB-associated lysosomal biogenesis, thus further enhancing lysosomal sunitinib entrapment and $\mathrm{MDR}^{[49]}$. A different study found that exposure of $786-\mathrm{O}$ renal cancer cells and HT-29 colorectal cancer to various tyrosine kinase inhibitors increased the number of lysosomes ${ }^{[64]}$. Taken together, these data lead credence to the notion that lysosomal drug sequestration induces lysosomal stress and TFEBmediated lysosomal biogenesis. The number of lysosomes in cancerous cells may prove to be an important consideration when selecting treatment options for cancer patients. A possible solution may be to target lysosomal biogenesis by finding ways to circumvent the nuclear translocation of TFEB. Support for this comes from a study that found that interaction of TFEB with active rag heterodimers promoted recruitment of TFEB to lysosomes, leading to mTORC1-dependent phosphorylation and inhibition of TFEB ${ }^{[65]}$.

\section{Lysosomal sequestration mediated by ATP-binding cassette transporter proteins}

Another mechanism by which lysosomes participate in drug sequestration involves ATP-binding cassette transporters (ABC-transporters; See Figure 1).

P-gp is often a representative $\mathrm{ABC}$-transporter, present in many malignant cells and a molecular target in cancer therapies ${ }^{[66,67]}$. P-gp is located on cell membranes. However, since P-gp expression also exists on the lysosomal membrane, lysosomal P-gp can transport cytotoxic agents into lysosomes. Support for this notion comes from reports that chemotherapeutics that are P-gp substrates and ionize at lysosomal $\mathrm{pH}(\mathrm{pH}$ 5), such as doxorubicin, danorubicin, vinblastine, and imatinib become localized and trapped inside lysosomes ${ }^{[57,68]}$. 


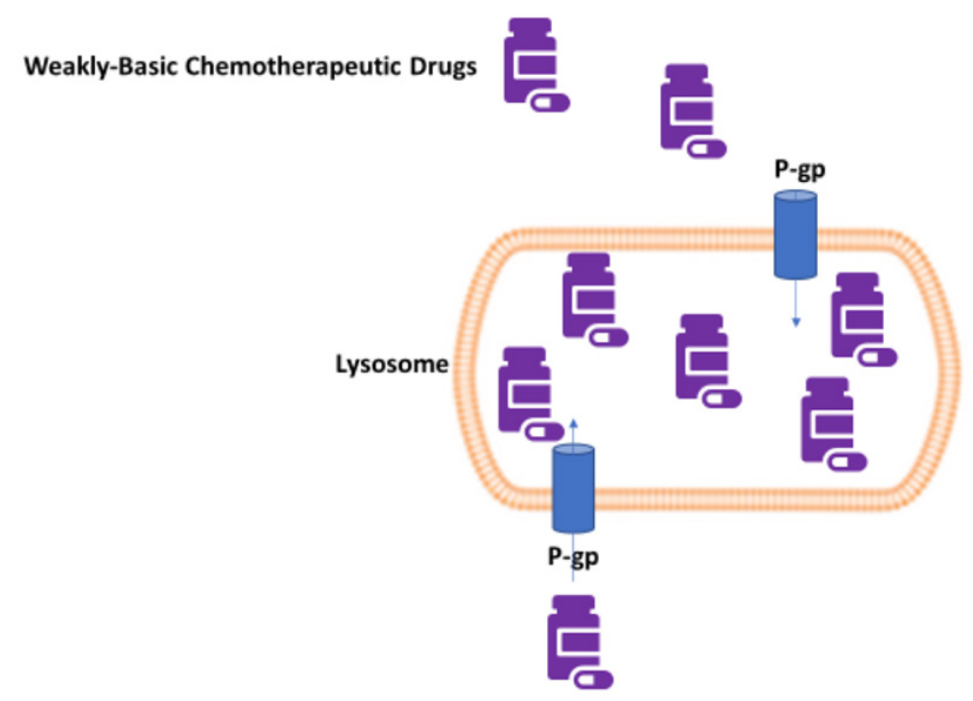

Figure 1. Lysosomal drug sequestration (LDS). P-gp expressed on lysosomal membranes contributes effluxes weakly-basic chemotherapeutics from the cytosol into the lysosomal lumen. LDS will decrease the cytosolic concentration of the drugs and their availability to molecular targets. P-gp: permeability-glycoprotein

The $\mathrm{pH}$ difference between the cytosol and lysosomal lumen is the driving force for lysosomal drug sequestration and is regulated by proton-pumping vacuolar-ATPase proteins ${ }^{[69]}$. This mechanism prevents such drugs from reaching their pharmacologic cytosolic concentrations and contribute to survival of the tumor cells. Lysosomal P-gp-mediated MDR can be overcome by using specific P-gp inhibitors or a combination of lysosomotropic agents with anticancer drugs. Support for this comes from work by Shiraishi et al ${ }^{[70]}$ (1986) demonstrating that chloroquine partially reversed the resistance of multi-drugresistant $\mathrm{KB}$ carcinoma cells to the P-gp substrates adriamycin, daunomycin, vincristine, vinblastine and actinomycin D. Furthermore, it has been shown that P-gp inhibitors valspodar and elacridar or silencing P-gp with siRNA reversed lysosomal sequestration of doxorubicin, leading to its redistribution to its intended target, the nucleus ${ }^{[6]}$. A recent report demonstrated that cell-surface P-gp is degraded by the lysosomal pathway and suggests that this pathway could be exploited to induce cell death in P-gp expressing tumors $^{[7]]}$. Lastly, lysosomal P-gp mediated resistance to sorafenib was reversed in hepatocellular carcinoma cells that were incubated with verapamil after drug pre-incubation ${ }^{[52]}$. However, the majority of clinical trials using P-gp inhibitors to suppress drug resistance have failed to show improved survival or remission rates ${ }^{[72]}$.

Other transporter proteins have also been implicated in promoting lysosomal drug sequestration. The ABC transporter $\mathrm{A} 3$ ( $\mathrm{ABCA3}$ ) was shown to contribute to lysosomal sequestration of imatinib and to potentiate resistance to this drug ${ }^{[73]}$. Indeed, the majority of the intracellular concentration of imatinib was found not in the cytosol, rather it was localized to lysosomes ${ }^{[73]}$. An additional obstacle is the fact that ABCA3 mediated resistance is correlated with an increase in lysosomal-related organelles ${ }^{[73]}$.

\section{Lysosomal exocytosis potentiates MDR}

Lysosomal exocytosis promotes MDR in malignant cells. Lysosomal exocytosis is a $\mathrm{Ca}^{2+}$-dependent process whereby lysosomes fuse with the plasma membrane and release their contents to the extracellular space ${ }^{[74]}$. Interestingly, lysosomal exocytosis is also regulated by TFEB and overexpression of TFEB is correlated with increased exocytosis ${ }^{[75]}$. It has been hypothesized that exocytosis of lysosomal sequestered drugs is another mechanism that contributes to reducing the concentration and efficacy of these drugs. Support for this comes from a study that found lysosomal exocytosis triggered in murine macrophages by treatment with agents that induced lysosomal alkalization ${ }^{[76]}$. These data lead credence to the notion that drugs that are sequestered in lysosomes are not trapped there indefinitely and are extruded via lysosomal exocytosis. 


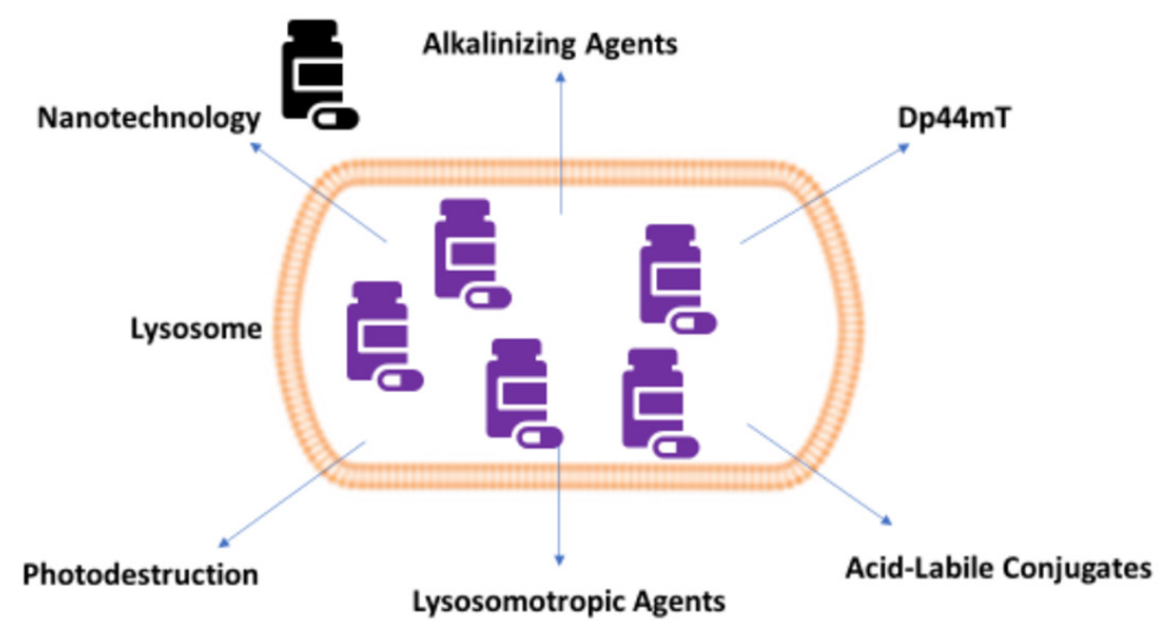

Figure 2. Putative strategies that may reverse lysosomal drug sequestration. Alkalinizing agents, nanotechnology, Dp $44 \mathrm{mT}$ photodestruction, lysosomotropic agents, and acid-labile conjugates may be employed to bypass lysosomal-mediated drug resistance. The purple medicine bottles represent chemotherapeutic drugs that are trapped in the lysosomal lumen and the black medicine bottle represents drugs that presumably will be sent back into the cytosol. Dp44mT: di-2-pyridylketone 4,4-dimethyl-3-thiosemicarbazone

Furthermore, lysosomal exocytosis of cathepsins B, D, K, and L have been shown to promote cell motility, angiogenesis, and metastasis ${ }^{[7,77]}$. Cathepsin D stimulates mitogen activated protein kinase signaling and angiogenic gene expression ${ }^{[77]}$.

\section{APPROACHES TO REVERSE LYSOSOMAL SEQUESTRATION}

\section{Alkalinizing agents}

We hypothesize that several mechanisms may reverse lysosomal drug sequestration. In the section below, we discuss putative strategies that might enable drugs that are trapped in lysosomes to translocate from lysosomal lumen to cytosol [Figure 2]. This would presumably aid in increasing drug availability to target sites.

Disrupting the acidification of lysosomes in multidrug-resistant cells has been shown to sensitize them to chemotherapeutics. A possible mechanism to reverse lysosomal drug accumulation of weak chemotherapeutic bases is by treatment with lysosome alkalinizing agents such as bafilomycin A1, a vesicular $\mathrm{H}^{+}$-ATPase inhibitor ${ }^{[78]}$. Bafilomycin $\mathrm{A} 1$, however, is too toxic for in vivo use and a more appropriate alkalinizing agent is chloroquine. One study administered chloroquine to mice and inhibited lysosomal function by raising the lysosomal $\mathrm{pH}^{[79]}$. A different study found that chloroquine potentiates the cytotoxic effects of doxorubicin in liver carcinoma cells ${ }^{[80]}$. Similarly, treatment of lysosomes in resistant cells with monensin, bafilomycin A1, or concanamycin A was sufficient to change the distribution of adriamycin to mimic that of drug-sensitive cells ${ }^{[47]}$. Additionally, prevention of subcellular trapping of nintedanib by lysosomal alkalinization abolished drug resistance ${ }^{[48]}$. These approaches appear plausible because $\mathrm{pH}$ gradient differences exist between MDR cancer cells and their wild-type drug sensitive counterpart cell lines ${ }^{[81]}$. These findings suggest that the use of well-tolerated alkalinizing agents may circumvent lysosomal drug sequestration and thereby increase cytotoxic drug efficacy.

\section{Lysosomotropic agents}

Another approach to abolish lysosomal drug sequestration is by using lipophilic drugs that become scavenged in lysosomes yet can induce $\mathrm{LMP}^{[70]}$. Chloroquine has been reported to promote cytotoxicity and to act synergistically with chemotherapeutic drugs. Chloroquine is a lysosomotropic agent that triggers destabilization of the lysosomal membrane in various tumor cells. In one study chloroquine was used to restore sensitivity to cisplatin in refractory non-small-cell lung cancer cells ${ }^{[82]}$. In another report, chloroquine was shown 
to potentiate the cytotoxic effects of topotecan by inhibiting autophagy ${ }^{[83]}$. The thiosemicarbazone, di-2pyridylketone 4,4-dimethyl-3-thiosemicarbazone (Dp44mT), has been shown to accumulate in lysosomes of tumor cells where it induces LMP ${ }^{[84]}$. Once in the lysosome, Dp44mT binds to copper forming a complex capable of producing cytotoxic reactive oxygen species (ROS) which triggers $\operatorname{LMP}^{[84]}$.

\section{Use of conjugates}

Conjugation of acid-labile chemicals to chemotherapeutic drugs has also been utilized to overcome lysosomal drug sequestration. Hydrazone is a commonly used linker molecule for this purpose because of its stability at cytosolic $\mathrm{pH}$ and its ability to hydrolyze at lysosomal $\mathrm{pH}^{[85,86]}$. Evidence for this comes from a report that conjugated doxorubicin to polyamidoamine dendrimers via hydrazone ${ }^{[87]}$. This complex was shown to release doxorubicin to the nucleus and induce cell death ${ }^{[87]}$. Additionally, degradable peptides, which are digested by lysosomal hydrolases, have been conjugated to cancer drugs and overcame lysosomal trapping $^{[88]}$.

\section{Photodestruction}

Lysosomal photodestruction of weakly basic chemotherapeutics that are also fluorochromes is another approach to reverse lysosomal sequestration. Photodestruction of imidazoacridinone-loaded lysosomes in MDR cancer cells resulted in cell lysis via formation of $\operatorname{ROS}^{[26]}$. Another study combined sunitinib with phototherapy to combat lysosomal localization of the drug ${ }^{[89]}$. However, this approach is of limited value because of the superficial and local treatment options of phototherapy.

\section{Dp44mT}

A recent study investigated the effects of glucose availability in cancer cells on reversing Pg-induced lysosomal drug entrapment. Seebacher and co-workers demonstrated that the anti-tumor agent and P-gp substrate Dp44mT induces LMP rather than lysosomal sequestration in response to glucose-induced stress ${ }^{[90]}$. When tumor cells are exposed to cellular stress, they produce increased amounts of ROS resulting in upregulation of P-gp expression ${ }^{[91]}$. As a result, P-gp actively pumps Dp44mT into lysosomes where it binds to copper, thereby forming ROS and triggering lysosomal membrane destabilization and apoptosis ${ }^{[2]}$. Lysosomes in neoplastic cells presumably have higher concentrations of copper compared to normal cells due to their increased requirement for metals ${ }^{[93]}$. The higher levels of copper are presumably needed by tumors for angiogenesis and metastasis ${ }^{[94,95]}$. Moreover, P-gp inhibitors such as elacridar abolished LMP induced by $\mathrm{Dp} 44 \mathrm{mT}^{[92]}$. These findings suggest that $\mathrm{Dp} 44 \mathrm{mT}$ only uses P-gp and not other $\mathrm{ABC}$ transporter proteins to overcome lysosomal drug sequestration. Taken together these results indicate that the design of novel metal-binding, P-gp substrate drugs like Dp44mT may be used to treat multidrug resistant tumors by targeting lysosomes.

\section{Nanotechnology}

Nanomedicine is another mechanism that shows promising results to overcome P-gp mediated MDR. A study showed that doxorubicin-loaded nanospheres (DOX-NS) evaded MDR and delivered a high concentration of the drug to the nucleus and cytosol ${ }^{[96]}$. This finding suggests that DOX-NS was not recognized as a P-gp substrate. Another study added a monoclonal antibody $2 \mathrm{C} 5$, which recognizes various tumor cells via tumor cell surface-bound nucleosomes, to doxorubicin liposomes ${ }^{[97]}$. The antibodydoxorubicin liposome complex significantly induced nuclear accumulation and cytotoxicity of doxorubicin in a doxorubicin-resistant colon cancer cell line ${ }^{[97]}$.

\section{CONCLUSION}

After long-term treatment with weakly basic anticancer drugs, lysosomal drug sequestration can occur. Lysosomes are attractive subcellular targets for creation of novel anticancer treatments for the following 
reasons. Tumor cells have larger, more active lysosomes, which make them more susceptible to lysosomal membrane degradation compared to lysosomes in non-neoplastic cells ${ }^{[98]}$. Additionally, cancer cells display higher metabolic rates and turnover of iron-containing proteins that sensitize them to ROS-induced $\mathrm{LMP}^{[99]}$. A novel imaging technique that can stably track lysosomes for at least $120 \mathrm{~h}$, irrespective of $\mathrm{pH}$ changes in the organelle, is now available and can be used to monitor lysosomes in cancer cells ${ }^{[100]}$. This new lysosomal tracing method is desirable over conventional acidotropic probes, which tend to dissipate in stressed lysosomes. The above characteristics of lysosomes in cancer cells should be fully exploited to trigger lysosomal-mediated cell death [Figure 1].

We have reviewed the important role played by lysosomes in MDR by three mechanisms: (1) lysosomal entrapment of weakly basic chemotherapeutics; (2) P-gp-mediated lysosomal sequestration; and (3) lysosomal exocytosis of anticancer drugs. Lysosomal potentiation of MDR is an issue that is compounded by the following factors. As described above, several of the weakly basic chemotherapeutic drugs are also P-gp substrates that are involved in P-gp-mediated lysosomal entrapment. Upregulated biogenesis of lysosomes in cancer cells leads to enlargement of the lysosomal compartment ${ }^{[101]}$. The enlarged compartment allows for a significant amount of drug to be scavenged from sites of action. Evidence for this comes from studies that demonstrated that drugs that localize to lysosomes can reach intracellular concentrations that are markedly higher than drug concentrations in the surrounding medium ${ }^{[102,103]}$. Similarly, lysosomal exocytosis of drugs reduces their intracellular concentrations and cytotoxic effects. There is a dire need to develop new strategies to overcome MDR in cancer treatment. Lysosomes, with acid hydrolases that can trigger the intrinsic apoptotic pathway and trigger caspase activation, serve as attractive targets for novel anticancer treatment modalities ${ }^{[104]}$. Specifically, the lysosomes of tumor cells exhibit alterations that are not observed in normal cells: increased cathepsin activity, shifts in different endolysosomal populations, and modified lysosomal trafficking $^{[14]}$. Further studies are warranted to fully exploit the unique differences in cancer lysosomes compared to their normal cell counterparts to sensitize tumor cells to cell death. The results of such projects should provide more effective strategies to bypass lysosomal-mediated drug resistance.

\section{DECLARATIONS}

Authors' contributions

Halaby R contributed solely to the article.

\section{Availability of data and materials}

Not applicable.

\section{Financial support and sponsorship}

None.

\section{Conflicts of interest}

The author declared that there are no conflicts of interest.

\section{Ethical approval and consent to participate}

Not applicable.

\section{Consent for publication}

Not applicable.

\section{Copyright}

(c) The Author(s) 2019. 


\section{REFERENCES}

1. de Duve C. The lysosome turns fifty. Nat Cell Biol 2005;7:847-9.

2. Guan JJ, Zhang XD, Sun W, Qi L, Wu JC, et al. DRAM1 regulates apoptosis through increasing protein levels and lysosomal localization of BAX. Cell Death Dis 2015;6:e1624.

3. Salvador N, Aguado C, Horst M, Knecht E. Import of a cytosolic protein into lysosomes by chaperone-mediated autophagy depends on its folding state. J Biol Chem 2000;275:27447-56.

4. Guillaumot P, Luquain C, Malek M, Huber AL, Brugière S, et al. Pdro, a protein associated with late endosomes and lysosomes and implicated in cellular cholesterol homeostasis. PLoS One 2010;5:e10977.

5. Kon S, Kobayashi N, Satake M. Altered trafficking of mutated growth factor receptors and their associated molecules: implication for human cancers. Cell Logist 2014;4:e28461.

6. Goh LK, Sorkin A. Endocytosis of receptor tyrosine kinases. Cold Spring Harb Perspect Biol 2013;5:a017459.

7. Mohamed MM, Sloane BF. Cysteine cathepsins: multifunctional enzymes in cancer. Nat Rev Cancer 2006; 6:764-75.

8. Vlodavsky I, Elkin M, Ilan N. Impact of heparanase and the tumor microenvironment on cancer metastasis and angiogenesis: basic aspects and clinical applications. Rambam Maimonides Med J 2011;2:e019.

9. Palermo C, Joyce JA. Cysteine cathepsin proteases as pharmacological targets in cancer. Trends Pharmacol Sci 2008;29:22-8.

10. Wang B, Sun J, Kitamoto S, Yang M, Grubb A, et al. Cathepsin S controls angiogenesis and tumor growth via matrix-derived angiogenic factors. J Biol Chem 2006; 281:6020-9.

11. Podgorski I, Linebaugh BE, Sameni M, Jedeszko C, Bhagat S, et al. Bone microenvironment modulates expression and activity of cathepsin B in prostate cancer. Neoplasia 2005;7:207-23.

12. Boya P, Andreau K, Poncet D, Zamzami N, Perfettini JL, et al. Lysosomal membrane permeabilization induces cell death in a mitochondrion-dependent fashion. J Exp Med 2003; 197:1323-34.

13. Kirkegaard T, Jäättelä M. Lysosomal involvement in cell death and cancer. Biochim Biophys Acta 2009;1793:746-54.

14. Fehrenbacher N, Jäättelä M. Lysosomes as targets for cancer therapy. Cancer Res 2005;65:2993-5.

15. Groth-Pedersen L, Jäättelä M. Combating apoptosis and multidrug resistant cancers by targeting lysosomes. Cancer Lett 2013; 332:265-74.

16. Holohan C, Van Schaeybroeck S, Longley DB, Johnston PG. Cancer drug resistance: an evolving paradigm. Nat Rev Cancer 2013;13:714-26.

17. Higgins CF. Multiple molecular mechanisms for multidrug resistance transporters. Nature 2007;446:749-57.

18. Al-Akra L, Bae DH, Sahni S, Huang MLH, Park KC, et al. Tumor stressors induce two mechanisms of intracellular P-glycoproteinmediated resistance that are overcome by lysosomal-targeted thiosemicarbazones. J Biol Chem 2018; 293:3562-87.

19. Rajagopal A, Simon SM. Subcellular localization and activity of multidrug resistance proteins. Mol Biol Cell 2003;14:3389-99.

20. Kunjachan S, Rychlik B, Storm G, Kiessling F, Lammers T. Multidrug resistance: Physiological principles and nanomedical solutions. Adv Drug Deliv Rev 2013;65:1852-65.

21. Shapira A, Livney YD, Broxterman HJ, Assaraf YG. Nanomedicine for targeted cancer therapy: towards the overcoming of drug resistance. Drug Resist Updat 2011;14:150-63.

22. Goldman SDB, Funk RS, Rajewski RA, Krise JP. Mechanisms of amine accumulation in, and egress from, lysosomes. Bioanalysis 2009;1:1445-59.

23. Kazmi F, Hensley T, Pope C, Funk RS, Loewen GJ, et al. Lysosomal sequestration (trapping) of lipophilic amine (cationic amphiphilic) drugs in immortalized human hepatocytes (Fa2N-4 cells). Drug Metab Dispos 2013;41:897-905.

24. Gotink KJ, Broxterman HJ, Labots M, de Haas RR, Dekker H, et al. Lysosomal sequestration of sunitinib: a novel mechanism of drug resistance. Clin Cancer Res 2011;17:7337-46.

25. Hurwitz SJ, Terashima M, Mizunuma N, Slapak CA. Vesicular anthracycline accumulation in doxorubicin-selected U-937 cells: participation of lysosomes. Blood 1997;89:3745-54

26. Adar Y, Stark M, Bram EE, Nowak-Sliwinska P, van den Bergh H, et al. Imidazoacridinone-dependent lysosomal photodestruction: a pharmacological Trojan horse approach to eradicate multidrug-resistant cancers. Cell Death Dis 2012;3:e293.

27. Herlevsen M, Oxford G, Owens CR, Conaway M, Theodorescu D. Depletion of major vault protein increases doxorubicin sensitivity and nuclear accumulation and disrupts its sequestration in lysosomes. Mol Cancer Ther 2007;6:1804-13.

28. Ndolo RA, Luan Y, Duan S, Forrest ML, Krise JP. Lysosomotropic properties of weakly basic anticancer agents promote cancer cell selectivity in vitro. PLoS One 2012;7:e49366.

29. Marshall LA, Rhee MS, Hofmann L, Khodjakov A, Schneider E. Increased lysosomal uptake of methotrexate-polyglutamates in two methotrexate-resistant cell lines with distinct mechanisms of resistance. Biochem Pharmacol 2005;71:203-13.

30. Jansen G, Barr H, Kathmann I, Bunni MA, Priest DG, et al. Multiple mechanisms of resistance to polyglutamatable and lipophilic antifolates in mammalian cells: role of increased folylpolyglutamylation,expanded folate pools, and intralysosomal drug sequestration. Mol Pharmacol 1999;55:761-9.

31. Li W, Yuan X, Nordgren G, Dalen H, Dubowchik GM, et al. Induction of cell death by the lysosomotropic detergent MSDH. FEBS Lett 2000;470:35-9.

32. Cirman T, Oresić K, Mazovec GD, Turk V, Reed JC, et al. Selective disruption of lysosomes in HeLa cells triggers apoptosis mediated by cleavage of Bid by multiple papain-like lysosomal cathepsins. J Biol Chem 2004;279:3578-87.

33. Kågedal K, Zhao M, Svensson I, Brunk UT. Sphingosine-induced apoptosis is dependent on lysosomal proteases. Biochem J 2001;359:335-43.

34. Erdal H, Berndtsson M, Castro J, Brunk U, Shoshan MC, et al. Induction of lysosomal membrane permeabilization by compounds that activate p53-independent apoptosis. Proc Natl Acad Sci USA 2005;102:192-7. 
35. Ostenfeld MS, Høyer-Hansen M, Bastholm L, Fehrenbacher N, Olsen OD, et al. Anti-cancer agent siramesine is a lysosomotropic detergent that induces cytoprotective autophagosome accumulation. Autophagy 2008;4:487-99.

36. Groth-Pedersen L, Ostenfeld MS, Høyer-Hansen M, Nylandsted J, Jäättelä M. Vincristine induces dramatic lysosomal changes and sensitizes cancer cells to lysosome-destabilizing siramesine. Cancer Res 2007;67:2217-25.

37. Rammer P, Groth-Pedersen L, Kirkegaard T, Daugaard M, Rytter A, et al. BAMLET activates a lysosomal cell death program in cancer cells. Mol Cancer Ther 2010;9:24-32.

38. Hahne JC, Kurz A, Meyer SR, Dietl J, Engel JB, et al. Anti-tumour activity of phosphoinositide-3-kinase antagonist AEZS-126 in models of ovarian cancer. Arch Gynecol Obstet 2015;291:131-41.

39. Yi YW, Kang HJ, Kim HJ, Hwang JS, Wang A, et al. Inhibition of constitutively activated phosphoinositide 3-kinase/AKT pathway enhances antitumor activity of chemotherapeutic agents in breast cancer susceptibility gene 1-defective breast cancer cells. Mol Carcinog 2013;52:667-75.

40. Martin-Fernandez C, Bales J, Hodgkinson C, Welman A, Welham MJ, et al. Blocking phosphoinositide 3-kinase activity in colorectal cancer cells reduces proliferation but does not increase apoptosis alone or in combination with cytotoxic drugs. Mol Cancer Res 2009;7:955-65.

41. Mousavi SA, Brech A, Berg T, Kjeken R. Phosphoinositide 3-kinase regulates maturation of lysosomes in rat hepatocytes. Biochem J 2003;372:861-9.

42. Silverman GA, Bartuski AJ, Cataltepe S, Gornstein ER, Kamachi Y, et al. SCCA1 and SCCA2 are proteinase inhibitors that map to the serpin cluster at 18q21.3. Tumour Biol 1998;19:480-7.

43. Suminami Y, Nawata S, Kato H. Biological role of SCC antigen. Tumour Biol 1998;19:488-93.

44. Kirkegaard T, Roth AG, Petersen NH, Mahalka AK, Olsen OD, et al. Hsp70 stabilizes lysosomes and reverts Niemann-Pick diseaseassociated lysosomal pathology. Nature 2010;463:549-53.

45. Nylandsted J, Gyrd-Hansen M, Danielewicz A, Fehrenbacher N, Lademann U, et al. Heat shock protein 70 promotes cell survival by inhibiting lysosomal membrane permeabilization. J Exp Med 2004;200:425-35.

46. Gyrd-Hansen M, Nylandsted J, Jäättelä M. Heat shock protein 70 promotes cancer cell viability by safeguarding lysosomal integrity. Cell Cycle 2004;3:1484-5.

47. Altan N, Chen Y, Schindler M, Simon SM. Defective acidification in human breast tumor cells and implications for chemotherapy. J Exp Med 1998;187:1583-98.

48. Englinger B, Kallus S, Senkiv J, Heilos D, Gabler L, et al. Intrinsic fluorescence of the clinically approved multikinase inhibitor nintedanib reveals lysosomal sequestration as resistance mechanism in FGFR-driven lung cancer. J Exp Clin Cancer Res 2017;36:122.

49. Zhitomirsky B, Assaraf YG. Lysosomal sequestration of hydrophobic weak base chemotherapeutics triggers lysosomal biogenesis and lysosome-dependent cancer multidrug resistance. Oncotarget 2015;6:1143-56.

50. Mayer LD, Bally MB, Cullis PR. Uptake of adriamycin into large unilamellar vesicles in response to a pH gradient. Biochim Biophys Acta 1986;857:123-6.

51. Angus DW, Baker JA, Mason R, Martin IJ. The potential influence of $\mathrm{CO} 2$, as an agent for euthanasia, on the pharmacokinetics of basic compounds in rodents. Drug Metab Dispos 2008;36:375-9.

52. Colombo F, Trombetta E, Cetrangolo P, Maggioni M, Razini P, et al. Giant lysosomes as a chemotherapy resistance mechanism in hepatocellular carcinoma cells. PLoS One 2014;9:e114787.

53. Reck M, Mellemgaard A, von Pawel J, Gottfried M, Bondarenko I, et al. Anti-angiogenic-specific adverse events in patients with nonsmall cell lung cancer treated with nintedanib and docetaxel. Lung Cancer 2015;90:267-73.

54. Mross K, Büchert M, Frost A, Medinger M, Stopfer P, et al. Vascular effects, efficacy and safety of nintedanib in patients with advanced, refractory colorectal cancer: a prospective phase I subanalysis. BMC Cancer 2014;14:510.

55. Molife LR, Omlin A, Jones RJ, Karavasilis V, Bloomfield D, et al. Randomized phase II trial of nintedanib, afatinib and sequential combination in castration-resistant prostate cancer. Future Oncol 2014;10:219-31.

56. Kutluk Cenik B, Ostapoff KT, Gerber DE, Brekken RA. BIBF 1120 (nintedanib), a triple angiokinase inhibitor, induces hypoxia but not EMT and blocks progression of preclinical models of lung and pancreatic cancer. Mol Cancer Ther 2013;12:992-1001.

57. Burger H, den Dekker AT, Segeletz S, Boersma AWM, de Bruijn P, et al. Lysosomal sequestration determines intracellular imatinib levels. Mol Pharmacol 2015;88:477-87.

58. Kalayda GV, Wagner CH, Buss I, Reedijk J, Jaehde U. Altered localisation of the copper efflux transporters ATP7A and ATP7B associated with cisplatin resistance in human ovarian carcinoma cells. BMC Cancer 2008;8:175.

59. Safaei R, Larson BJ, Cheng TC, Gibson MA, Otani S, et al. Abnormal lysosomal trafficking and enhanced exosomal export of cisplatin in drug-resistant human ovarian carcinoma cells. Mol Cancer Ther 2005;4:1595-604.

60. Settembre C, Zoncu R, Medina DL, Vetrini F, Erdin S, et al. A lysosome-to-nucleus signalling mechanism senses and regulates the lysosome via mTOR and TFEB. EMBO J 2012;31:1095-108.

61. Martina JA, Chen Y, Gucek M, Puertollano R. MTORC1 functions as a transcriptional regulator of autophagy by preventing nuclear transport of TFEB. Autophagy 2012;8:903-14.

62. Medina DL, Di Paola S, Peluso I, Armani A, De Stefani D, et al. Lysosomal calcium signalling regulates autophagy through calcineurin and TFEB. Nat Cell Biol 2015;17:288-99.

63. Sardiello M, Palmieri M, di Ronza A, Medina DL, Valenza M, et al. A gene network regulating lysosomal biogenesis and function. Science 2009;325:473-7.

64. Gotink KJ, Rovithi M, de Haas RR, Honeywell RJ, Dekker H, et al. Cross-resistance to clinically used tyrosine kinase inhibitors sunitinib, sorafenib and pazopanib. Cell Oncol (Dordr) 2015;38:119-29. 
65. Martina JA, Puertollano R. Rag GTPases mediate amino acid-dependent recruitment of TFEB and MITF to lysosomes. J Cell Biol 2013;200:475-91.

66. Syed SB, Arya H, Fu IH, Yeh TK, Periyasamy L, et al. Targeting P-glycoprotein: investigation of piperine analogs for overcoming drug resistance in cancer. Sci Rep 2017;7:7972.

67. Sinha BK, Bortner CD, Mason RP, Cannon RE. Nitric oxide reverses drug resistance by inhibiting ATPase activity of p-glycoprotein in human multi-drug resistant cancer cells. Biochim Biophys Acta Gen Subj 2018;1862:2806-14.

68. Yamagishi T, Sahni S, Sharp DM, Arvind A, Jansson PJ, et al. P-glycoprotein mediates drug resistance via a novel mechanism involving lysosomal sequestration. J Biol Chem 2013;288:31761-71.

69. Forgac M. Vacuolar ATPases: rotary proton pumps in physiology and pathophysiology. Nat Rev Mol Cell Biol 2007;8:917-29.

70. Shiraishi N, Akiyama S, Kobayashi M, Kuwano M. Lysosomotropic agents reverse multiple drug resistance in human cancer cells. Cancer Lett 1986;30:251-9.

71. Katayama K, Kapoor K, Ohnuma S, Patel A, Swaim W, et al. Revealing the fate of cell surface human P-glycoprotein (ABCB1): the lysosomal degradation pathway. Biochim Biophys Acta 2015;1853:2361-70.

72. Palmeira A, Sousa E, Vasconcelos MH, Pinto MM. Three decades of P-gp inhibitors: skimming through several generations and scaffolds. Curr Med Chem 2012;19:1946-2025.

73. Chapuy B, Panse M, Radunski U, Koch R, Wenzel D, et al. ABC transporter A3 facilitates lysosomal sequestration of imatinib and modulates susceptibility of chronic myeloid leukemia cell lines to this drug. Haematologica 2009;94:1528-36.

74. Rodríguez A, Webster P, Ortego J, Andrews NW. Lysosomes behave as Ca2+-regulated exocytic vesicles in fibroblasts and epithelial cells. J Cell Biol 1997;137:93-104.

75. Medina DL, Fraldi A, Bouche V, Annunziata F, Mansueto G, et al. Transcriptional activation of lysosomal exocytosis promotes cellular clearance. Dev Cell 2011;21:421-30.

76. Sundler R. Lysosomal and cytosolic pH as regulators of exocytosis in mouse macrophages. Acta Physiol Scand 1997;161:553-6.

77. Kallunki T, Olsen OD, Jäättelä M. Cancer-associated lysosomal changes: friends or foes? Oncogene 2013;32:1995-2004.

78. Ouar Z, Bens M, Vignes C, Paulais M, Pringel C, et al. Inhibitors of vacuolar H+-ATPase impair the preferential accumulation of daunomycin in lysosomes and reverse the resistance to anthracyclines in drug-resistant renal epithelial cells. Biochem J 2003;370:185-93.

79. Gotink KJ, Broxterman HJ, Honeywell RJ, Dekker H, de Haas RR, et al. Acquired tumor cell resistance to sunitinib causes resistance in a HT-29 human colon cancer xenograft mouse model without affecting sunitinib biodistribution or the tumor microvasculature. Oncoscience 2014;1:844-53.

80. Li Y, Sun Y, Jing L, Wang J, Yan Y, et al. Lysosome inhibitors enhance the chemotherapeutic activity of doxorubicin in hepg2 cells. Chemotherapy 2017;62:85-93.

81. Wang E, Lee MD, Dunn KW. Lysosomal accumulation of drugs in drug-sensitive MES-SA but not multidrug-resistant MES-SA/Dx5 uterine sarcoma cells. J Cell Physiol 2000;184:263-74.

82. Circu M, Cardelli J, Barr MP, O’Byrne K, Mills G, et al. Modulating lysosomal function through lysosome membrane permeabilization or autophagy suppression restores sensitivity to cisplatin in refractory non-small-cell lung cancer cells. PLoS One 2017;12:e0184922.

83. Wang Y, Peng RQ, Li DD, Ding Y, Wu XQ, et al. Chloroquine enhances the cytotoxicity of topotecan by inhibiting autophagy in lung cancer cells. Chin J Cancer 2011;30:690-700.

84. Lovejoy DB, Jansson PJ, Brunk UT, Wong J, Ponka P, et al. Antitumor activity of metal-chelating compound Dp44mT is mediated by formation of a redox-active copper complex that accumulates in lysosomes. Cancer Res 2011;71:5871-80.

85. Yang X, Grailer JJ, Pilla S, Steeber DA, Gong S. Tumor-targeting, pH-responsive, and stable unimolecular micelles as drug nanocarriers for targeted cancer therapy. Bioconjug Chem 2010;21:496-504.

86. Ríhová B, Etrych T, Pechar M, Jelínková M, Stastný M, et al. Doxorubicin bound to a HPMA copolymer carrier through hydrazone bond is effective also in a cancer cell line with a limited content of lysosomes. J Control Release 2001;74:225-32.

87. Lai PS, Lou PJ, Peng CL, Pai CL, Yen WN, et al. Doxorubicin delivery by polyamidoamine dendrimer conjugation and photochemical internalization for cancer therapy. J Control Release 2007;122:39-46.

88. Minko T, Kopecková P, Pozharov V, Kopecek J. HPMA copolymer bound adriamycin overcomes MDR1 gene encoded resistance in a human ovarian carcinoma cell line. J Control Release 1998; 54:223-33.

89. Nowak-Sliwinska P, Weiss A, van Beijnum JR, Wong TJ, Kilarski WW, et al. Photoactivation of lysosomally sequestered sunitinib after angiostatic treatment causes vascular occlusion and enhances tumor growth inhibition. Cell Death Dis 2015;6:e1641.

90. Seebacher NA, Lane DJR, Jansson PJ, Richardson DR. Glucose modulation induces lysosome formation and increases lysosomotropic drug sequestration via the P-glycoprotein drug transporter. J Biol Chem 2016;291:3796-820.

91. Seebacher NA, Richardson DR, Jansson PJ. A mechanism for overcoming P-glycoprotein-mediated drug resistance: novel combination therapy that releases stored doxorubicin from lysosomes via lysosomal permeabilization using Dp44mT or DpC. Cell Death Dis 2016;7:e2510.

92. Jansson PJ, Yamagishi T, Arvind A, Seebacher N, Gutierrez E, et al. Di-2-pyridylketone 4,4-dimethyl-3-thiosemicarbazone (Dp44mT) overcomes multidrug resistance by a novel mechanism involving the hijacking of lysosomal P-glycoprotein (Pgp). J Biol Chem 2015;290:9588-603.

93. Weinberg ED. Iron withholding: a defense against infection and neoplasia. Physiol Rev 1984;64:65-102.

94. Xie H, Kang YJ. Role of copper in angiogenesis and its medicinal implications. Curr Med Chem 2009;16:1304-14.

95. Hassouneh B, Islam M, Nagel T, Pan Q, Merajver SD, et al. Tetrathiomolybdate promotes tumor necrosis and prevents distant metastases by suppressing angiogenesis in head and neck cancer. Mol Cancer Ther 2007;6:1039-45. 
96. Cuvier C, Roblot-Treupel L, Millot JM, Lizard G, Chevillard S, et al. Doxorubicin-loaded nanospheres bypass tumor cell multidrug resistance. Biochem Pharmacol 1992;44:509-17.

97. Elbayoumi TA, Torchilin VP. Enhanced cytotoxicity of monoclonal anticancer antibody 2C5-modified doxorubicin-loaded PEGylated liposomes against various tumor cell lines. Eur J Pharm Sci 2007;32:159-68.

98. Ono K, Kim SO, Han J. Susceptibility of lysosomes to rupture is a determinant for plasma membrane disruption in tumor necrosis factor alpha-induced cell death. Mol Cell Biol 2003;23:665-76.

99. Eaton JW, Qian M. Molecular bases of cellular iron toxicity. Free Radic Biol Med 2002;32:833-40.

100. Xue Z, Wang S, Li J, Chen X, Han J, et al. Bifunctional super-resolution imaging probe with acidity-independent lysosome-retention mechanism. Anal Chem 2018;90:11393-400.

101. Zhitomirsky B, Assaraf YG. Lysosomes as mediators of drug resistance in cancer. Drug Resist Updat 2016;24:23-33.

102. Kornhuber J, Henkel AW, Groemer TW, Städtler S, Welzel O, et al. Lipophilic cationic drugs increase the permeability of lysosomal membranes in a cell culture system. J Cell Physiol 2010;224:152-64.

103. Fu D, Zhou J, Zhu WS, Manley PW, Wang YK, et al. Imaging the intracellular distribution of tyrosine kinase inhibitors in living cells with quantitative hyperspectral stimulated Raman scattering. Nat Chem 2014;6:614-22.

104. Fehrenbacher N, Gyrd-Hansen M, Poulsen B, Felbor U, Kallunki T, et al. Sensitization to the lysosomal cell death pathway upon immortalization and transformation. Cancer Res 2004;64:5301-10. 